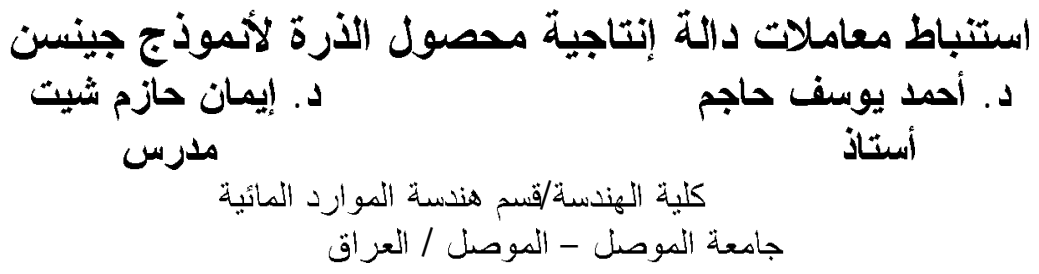

الخلاصة

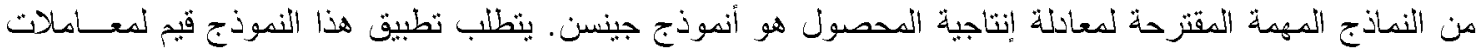

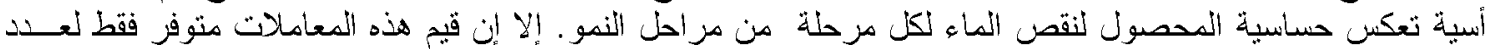

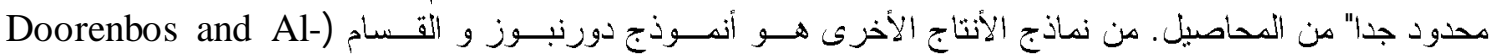

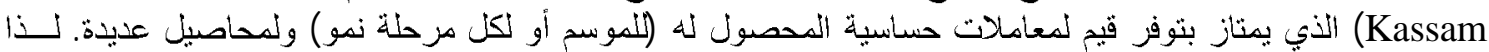

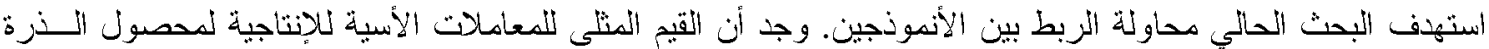

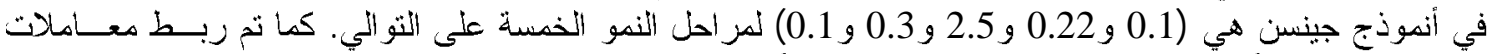

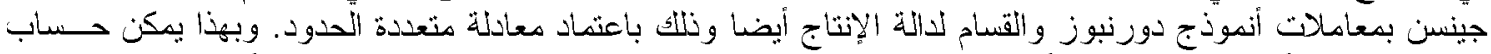

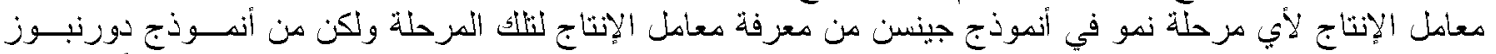

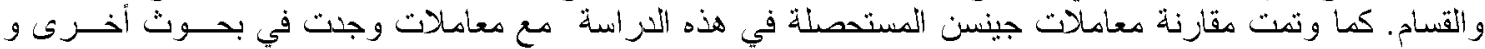
أعطت المقاز نة نتائج مقاربة. الكنسات المفتاحية: دالة إنتاجية المحصون، معاملات الإنتاجية، أندوذج جينسن، الذزة

\title{
Estimating Yield Response Factors for Maize Crop in Jensen Model
}

\author{
Dr. Ahmed Yousif Hachum Dr. Eman Hazim Sheet \\ Water Resources Department, \\ College of Engineering, University of Mosul , Mosul, Iraq
}

\begin{abstract}
Among the important proposed water-dependent crop production function is that of Jensen's. The application of this model requires knowledge of power parameters that reflect the sensitivity of each growth stage to water deficit. However, the values of these parameters are available only for a limited number of crops. Among the production models to which sensitivity parameters are quite available is that of Doorenbos and Al-Kassam. The objective of this study is to relate these two models. The results of the analysis showed that the yield response parameters for maize crop in Jensen model were found to be $(0.1,0.22,2.5,0.3,0.1)$ respectively. Also, a polynomial equation is developed to relate predicted yield response parameters in Jensen model to yield response factors, valid for all growth stages, in Doorenbos and Al-Kassam model. A comparison was made between the predicted parameters with those obtained from previous works indicating acceptable agreement.
\end{abstract}

Keywords: crop production function, yield response factor, Jensen model, maize. 


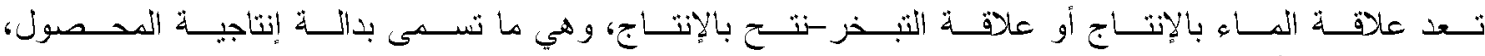

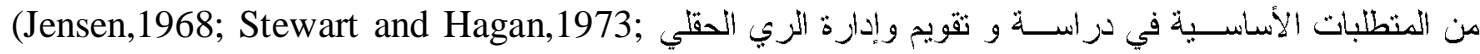

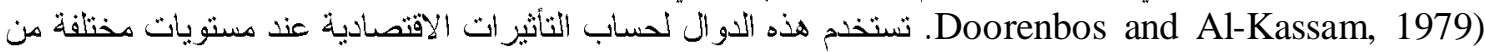
توفر المياه للمحصول. من أولى البحوث التي وضعت لتقنير معانلات الإنتاجية (1971) Yaron حيث وضع معادلات

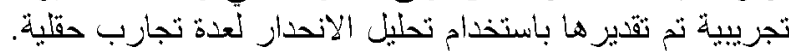

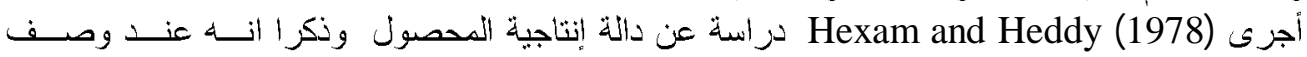

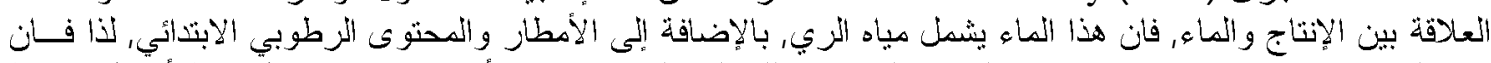

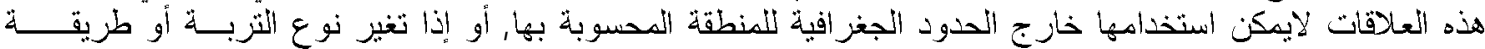

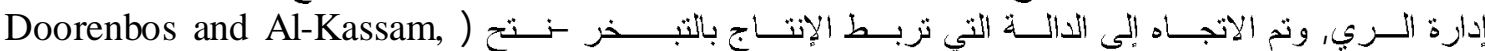

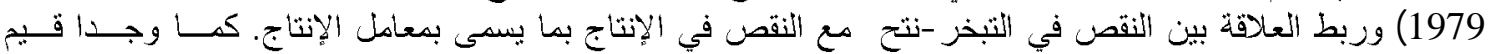

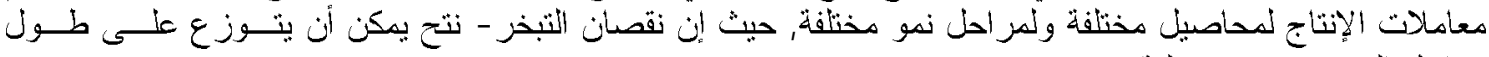

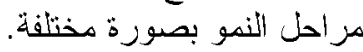

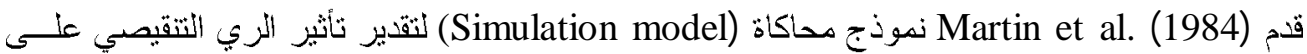

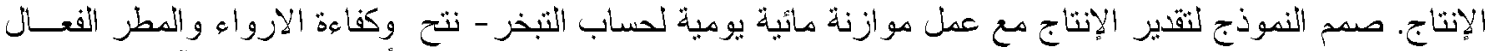

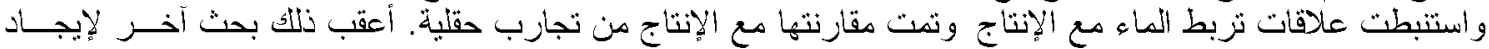

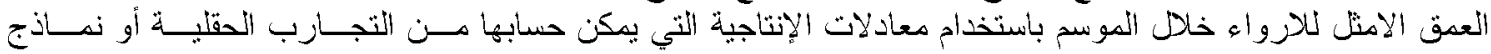

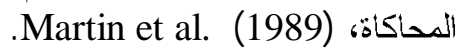

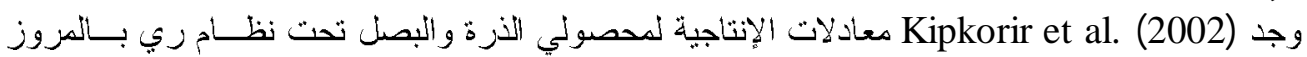

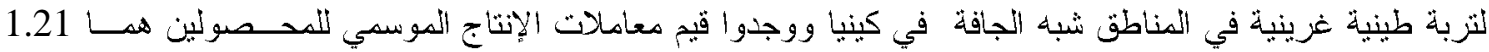

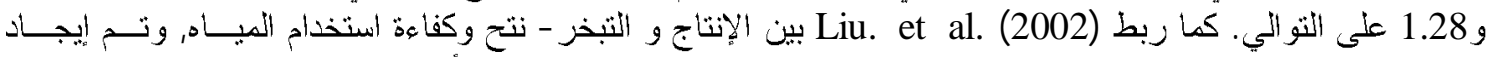

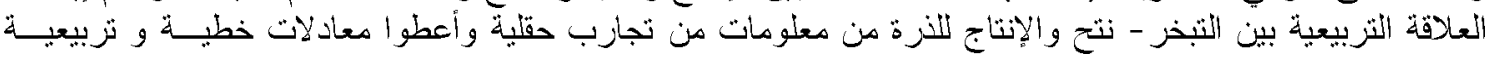

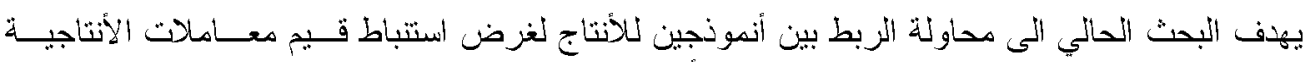
لربط المتغبرات.

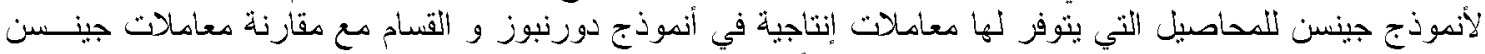

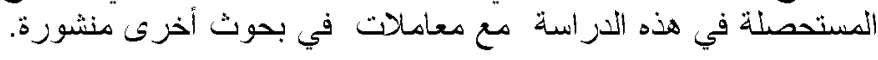

\section{طرق ومو اد البحث}

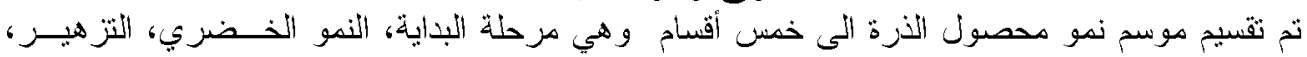

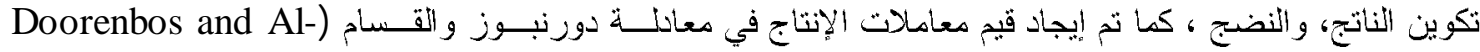
(Kassam, 1979

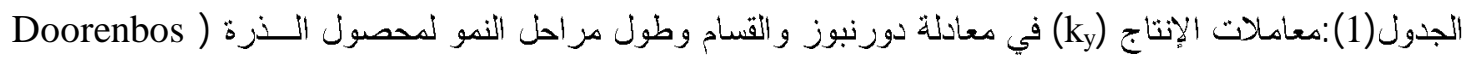
(and Al-Kassam, 1979

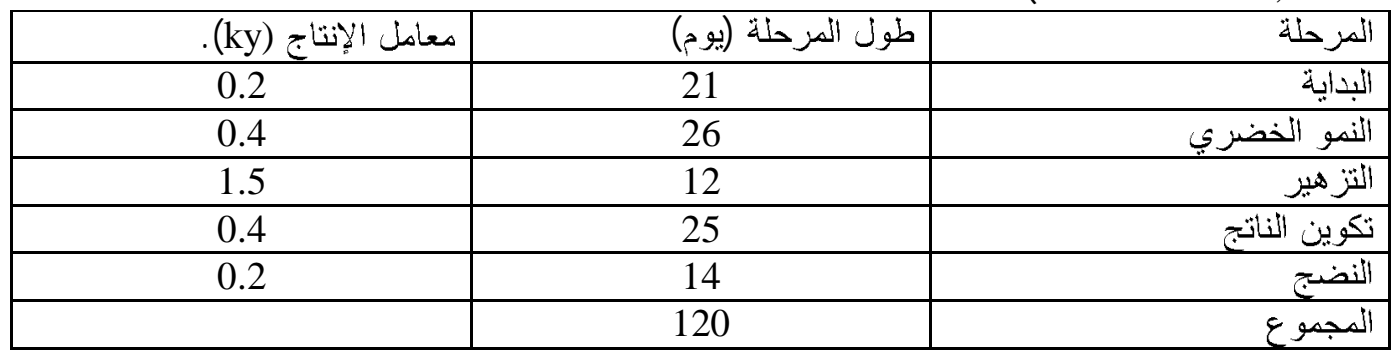




$$
Y / Y_{\max }=\prod_{j=1}^{n s}\left(A E T_{j} / C P E T_{j}\right)^{\lambda j}
$$

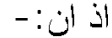

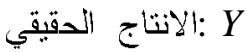

$$
\begin{aligned}
& \text { : : الانتاج الإقصى : } \\
& \text { AET }
\end{aligned}
$$

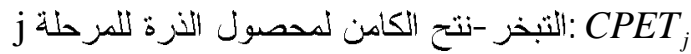

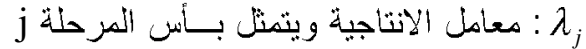

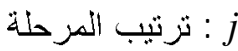

$$
\begin{aligned}
& 5 \text { : عد المر احن ويسأوي } 5
\end{aligned}
$$

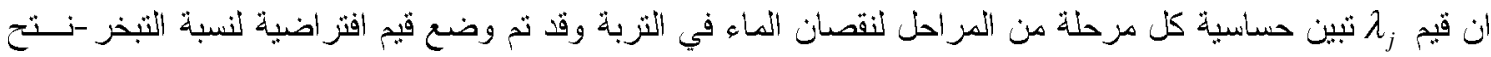
الحقيقي التى التبخر -نتح الكامن لمحصول التزة (

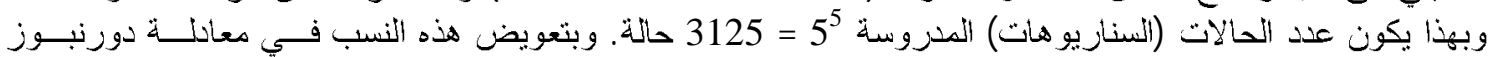

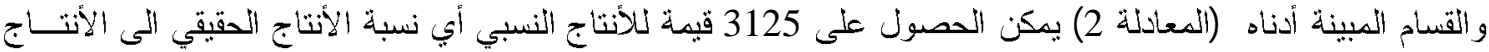

$$
\frac{Y}{Y_{\max }}=\prod_{j=1}^{n s}\left(1-k y_{j}\left(1-\frac{A E T_{j}}{C P E T_{j}}\right)\right)
$$

و التي فيها $k y_{j}$ يمنل معامل إنتاجية المحصون لكل مرحلة من مر احل النمو وهذه المعاملات متوفزة لعدد كبير من المحاصيل (Doorenbos and Al-Kassam, 1979) . أما

$$
\text { الأقصصى للمزحلة i ـ ولغزرض تقويم المعاملات }
$$

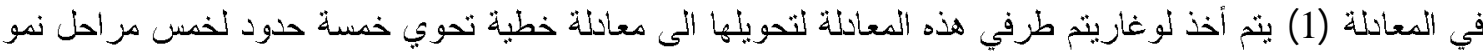
وكما مبين فيما يلي:

$$
Z=\lambda_{1} X_{1}+\lambda_{2} X_{2}+\lambda_{3} X_{3}+\lambda_{4} X_{4}+\lambda_{5} X_{5}
$$

$$
\begin{aligned}
& \text { حيث أن: } \\
& \text { = ل لوغاريتم } \\
& \text { لو لو اريتم }) \text { =X }
\end{aligned}
$$

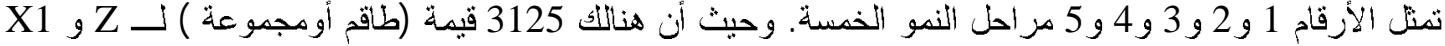

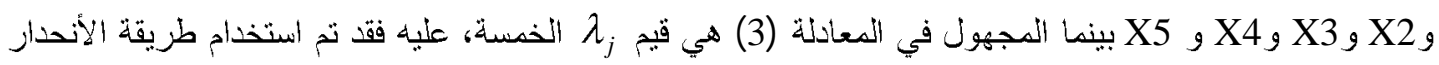

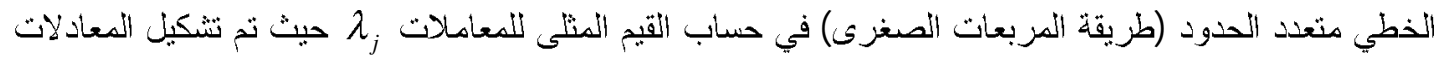

$$
\text { الخمس الآتية بخمس معاملات مجهو لنة: }
$$

$$
\begin{aligned}
& \sum Y_{i} X_{1 i}=\lambda_{1} \sum X_{1 i}^{2}+\lambda_{2} \sum X_{1 i} X_{2 i}+\lambda_{3} \sum X_{1 i} X_{3 i}+\lambda_{4} \sum X_{1 i} X_{4 i}+\lambda_{5} \sum X_{1 i} X_{5 i} \ldots \ldots \text { (4) } \\
& \sum Y_{i} X_{2 i}=\lambda_{1} \sum X_{1 i} X_{2 i}+\lambda_{2} \sum X_{2 i}^{2}+\lambda_{3} \sum X_{2 i} X_{3 i}+\lambda_{4} \sum X_{2 i} X_{4 i}+\lambda_{5} \sum X_{2 i} X_{5 i} \ldots \ldots \text { (5) } \\
& \sum Y_{i} X_{3 i}=\lambda_{1} \sum X_{1 i} X_{3 i}+\lambda_{2} \sum X_{2 i} X 3 i+\lambda_{3} \sum X_{3 i}^{2}+\lambda_{4} \sum X_{3 i} X_{4 i}+\lambda_{5} \sum X_{3 i} X_{5 i} \ldots \ldots \text { (6) }
\end{aligned}
$$




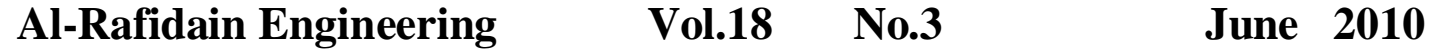

$\sum Y_{i} X_{4 i}=\lambda_{1} \sum X_{1 i} X_{4 i}+\lambda_{2} \sum X_{2 i} X_{4 i}+\lambda_{3} \sum X_{3 i} X_{4 i}+\lambda_{4} \sum X_{4 i}^{2}+\lambda_{5} \sum X_{4 i} X_{5 i}$
$\sum Y_{i} X_{5 i}=\lambda_{1} \sum X_{1 i} X_{5 i}+\lambda_{2} \sum X_{2 i} X_{5 i}+\lambda_{3} \sum X_{3 i} X_{5 i}+\lambda_{4} \sum X_{4 i} X_{5 i}+\lambda_{5} \sum X_{5 i}^{2}$ و التي يتغير فيها العداد $i$ من 1 الى 3125.

\section{النتائج و المناقثة}

بعد حساب قيم الكميات الحاوية على زمز الجمع $\sum$ نم الحصول على المعادلات الخمسة الخطية الآتية: $473.4=352.2 \lambda_{1}+200.4 \lambda_{2}+126.8 \lambda_{3}+200.4 \lambda_{4}+200.4 \lambda_{5}$

$493.2=200.4 \lambda_{1}+352.8 \lambda_{2}+126.8 \lambda_{3}+200.4 \lambda_{4}+200.4 \lambda_{5}$

$428.7=126.8 \lambda_{1}+126.8 \lambda_{2}+135.6 \lambda_{3}+126.8 \lambda_{4}+126.8 \lambda_{5}$

$505=200.4 \lambda_{1}+200.4 \lambda_{2}+126.8 \lambda_{3}+352.8 \lambda_{4}+200.4 \lambda_{5}$

$473.4=200.4 \lambda_{1}+200.4 \lambda_{2}+126.8 \lambda_{3}+200.4 \lambda_{4}+352.8 \lambda_{5}$

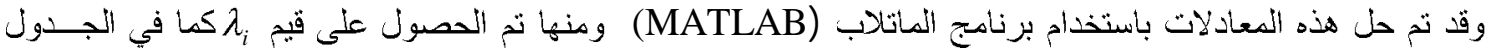

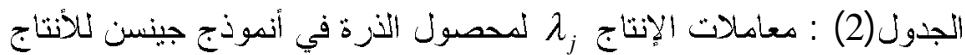

\begin{tabular}{|c|c|c|c|c|c|}
\hline 5 & 4 & 3 & 2 & 1 & المر حلة \\
\hline 0.1 & 0.3 & 2.5 & 0.22 & 0.1 & معامل الانتاج \\
\hline
\end{tabular}

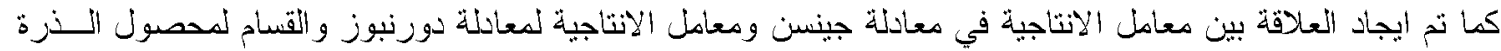
حيث تم ربط المتغبرين بمعادلة هتعددة الحدود وبمعامل ارتباط (0.992). $\lambda_{i}=0.9172 k y_{i}^{2}+0.29 k y_{i}$

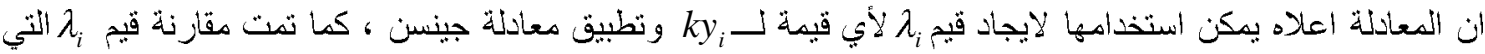

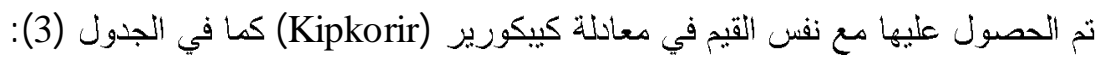

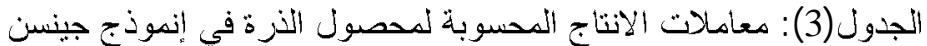

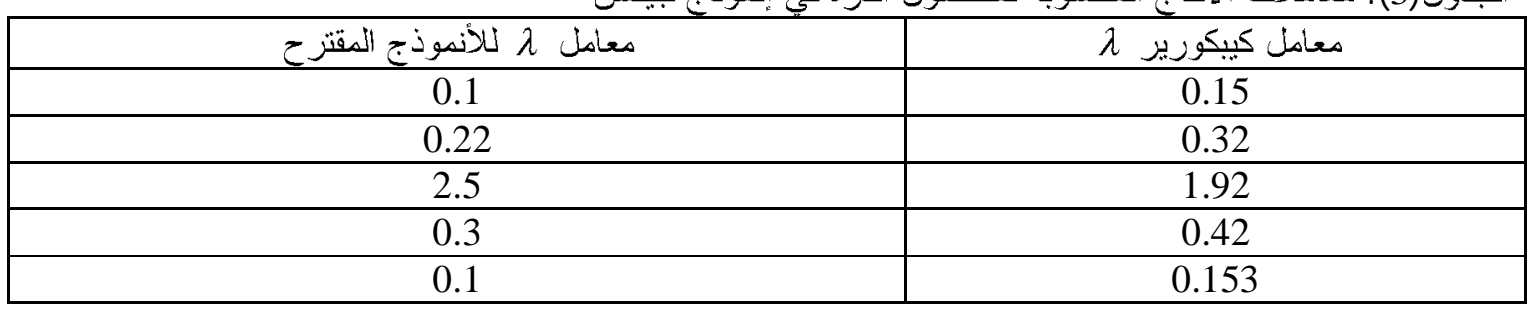

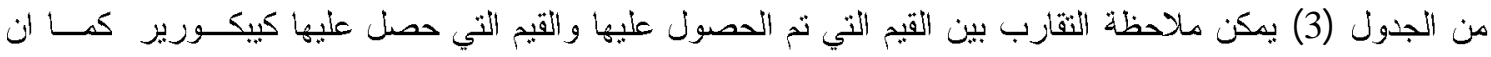

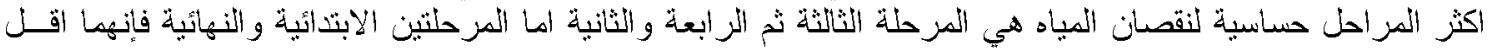

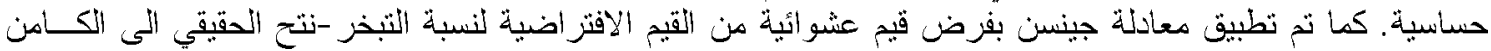

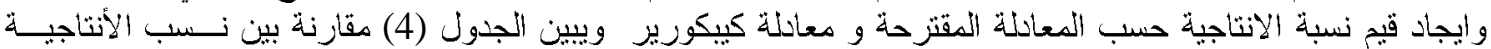

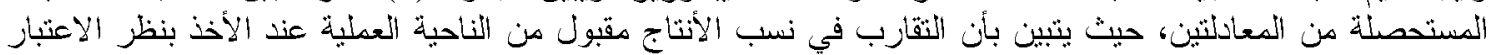

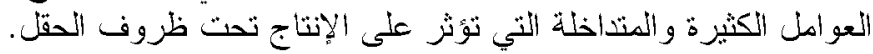

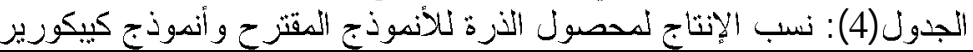

\begin{tabular}{|c|c|c|}
\hline $\mathrm{y} / \mathrm{ymax}$ & نسبة الأنتا|- & نسبة التتبخر -نتح الحقيقي التى التبخر - نتح الكامن \\
\hline 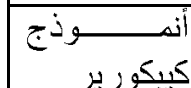 & ألأنـــــــوذج & المرحلة \\
\hline
\end{tabular}




\begin{tabular}{|c|c|c|c|c|c|c|}
\hline & & 5 & 4 & 3 & 2 & 1 \\
\hline 0.87 & 0.91 & 1.0 & 1.0 & 1.0 & 1.0 & 0.4 \\
\hline 0.74 & 0.82 & 1.0 & 1.0 & 1.0 & 0.4 & 1.0 \\
\hline 0.17 & 0.10 & 1.0 & 1.0 & 0.4 & 1.0 & 1.0 \\
\hline 0.68 & 0.76 & 1.0 & 0.4 & 1.0 & 1.0 & 1.0 \\
\hline 0.87 & 0.91 & 0.4 & 1.0 & 1.0 & 1.0 & 1.0 \\
\hline
\end{tabular}

\section{الثمدادر}

1. Doorenbos, J. and A. H. Kassam (1979). "Yield response to water". FAO Irrigation and Drainage Paper No. 33.

2. Hexam, R. W. and E. O. Heady (1978). "Water production function for irrigated agriculture". Enter for Agriculture and Rural Development, Iowa State University press, Ames, IA.

3. Jensen, M.E. (1968).“Water consumption by agriculture plants ".Water Deficits and Plant Growth, T T. Kozlowski, 1st ed., vol. 2,Academic press, New York, pp. 1-22.

4. Kipkorir, E. C. , D. Raes and B. Massawe (2002).”Seasonal water production functions and yield response factors for maize and onion in Perkerra, Kenya". Agricultural Water Management, 56: 229-240.

5. Liu, W. Z. , D. J. Hunsaker, Y. S. Li, X. Q. Xie, and G. W. Wall (2002). "Interrelations of yield, evapotranspiration, and water use efficiency from marginal analysis of water production functions". Agricultural Water Management, 56: 143-151.

6. Martin, D. L ., D. G. Watts and J. R. Jilley (1984). "Model and production function for irrigation management". Journal of the Irrigation and Drainage Division, ASCE 110(2): 149-164.

7. Martin, D. L. , J. R. Gilley and R. J. Supalla (1989). “Evaluation of irrigation planning decisions" Journal of the Irrigation and Drainage Division, ASCE, 115(1): 5877.

8. Stewart, J. I. and R. M. Hagan (1973). "Function to predict effects of crop water deficits". Journal of the Irrigation and Drainage Division, ASCE, 99(4): 421-439

9. Yaron, D. (1971). "Estimation and use of water production function in crops". Journal of the Irrigation and Drainage Division, ASCE, 97(2): 291-303.

$$
\text { تم اجر اء البحث في كلية الهنسة - جامعة الموصل }
$$

